\title{
Tolerância de Cana-de-Açúcar a Herbicidas Avaliada pela DifERENÇA DOS TRATAMENTOS ${ }^{1}$
}

\author{
Sugarcane Tolerance to Herbicides Based on the Difference between Treatments
}

\author{
SCHIAVETTO, A.R. ${ }^{2}$, PERECIN, D. ${ }^{3}$, AZANIA, C.A.M. ${ }^{4}$, ZERA, F.S. ${ }^{5}$, AZANIA, A.A.P.M. ${ }^{6}$ e \\ LORENZATO, C.M. ${ }^{7}$
}

\begin{abstract}
RESUMO - O objetivo deste trabalho foi avaliar a tolerância dos cultivares RB855453, RB845257, SP90-3414, SP90-1638, SP89-1115, SP81-3250, IAC91-2218 e IAC91-5155 de cana-de-açúcar em pós-emergência inicial quanto à aplicação dos herbicidas sulfentrazone + diuron + hexazinone, metsulfuron-methyl + sulfentrazone, diuron + hexazinone + clomazone, metribuzin + diuron + hexazinone, diuron + hexazinone + MSMA e ametryn + trifloxysulfuronsodium + diuron + hexazinone, utilizando-se da diferença com a testemunha pareada, considerando a minimização da área experimental. O experimento foi conduzido assumindo que as testemunhas pareadas ao respectivo tratamento facilitam a avaliação da tolerância dos cultivares aos tratamentos herbicidas. A instalação do experimento foi em blocos casualizados, utilizando-se o esquema fatorial 8 (cultivares) x 6 (herbicidas). As parcelas foram constituídas por seis linhas de cana-de-açúcar, sendo uma linha central destinada ao tratamento herbicida (TH) e outra à testemunha pareada (TP); as demais linhas foram usadas como bordaduras. Para verificar se as diferenças médias (TP-TH) não diferem de zero, utilizouse a estatística $\mathrm{t}=(\mathrm{TP}-\mathrm{TH}) /\left((\mathrm{QMRes} / \mathrm{n})^{1 / 2} \mathrm{ou}\right.$, de forma equivalente, a diferença mínima significativa com zero, dmsO(TP-TH) $=\left|\mathrm{t} /\left((\mathrm{QMRes} / \mathrm{n})^{1 / 2}\right)\right|$, em que QMRes é o quadrado médio da análise de variância (com 47 graus de liberdade e $\mathrm{p}<0,05, \mathrm{t}=2$ ) e $\mathrm{n}$ representa o número de repetições da respectiva média: 2 para comparações de herbicidas em um cultivar e 2 × $6=12$ para comparações da média geral de cultivares. Pela metodologia proposta, os cultivares foram tolerantes às associações de herbicidas sem prejuízo final da produtividade e da qualidade da matéria-prima, embora ocorram diferenças iniciais entre cultivares quanto a sintomas de intoxicação, teor de clorofila e altura das plantas.
\end{abstract}

Palavras-chave: seletividade, Saccharum spp., estatística, associação de herbicidas.

ABSTRACT - The objective of this work was to evaluate the tolerance of the sugarcane cultivars RB855453, RB845257, SP90-3414, SP90-1638, SP89-1115, SP81-3250, IAC91- 2218 and IAC91-5155 at early post-emergence after application of sulfentrazone + diuron + hexazinone; sulfentrazone + metsulfuron-methyl, diuron + hexazinone + clomazone; metribuzin + diuron + hexazinone, diuron + hexazinone + MSMA; and ametryn + trifloxysulfuron-sodium + diuron + hexazinone, using the difference with the paired control. It was assumed that the control paired to the respective treatments can facilitate the evaluation of the cultivars' tolerance to the herbicide treatments. The experiment was arranged in a randomized block design, using the factorial 8 (cultivars) $x 6$ (herbicides). The plots consisted of six rows of sugarcane, with a central line for the treatment herbicide (TH) and a matched control (TP), with the other lines being used as

1 Recebido para publicação em 1.4.2011 e aprovado em 28.9.2011.

Parte da Dissertação de mestrado da primeira autora.

2 Mestranda em Genética e Melhoramento de Plantas, Faculdade de Ciências Agrárias e Veterinárias, Universidade Estadual Paulista - UNESP, Via de Acesso Prof. Paulo Donato Castellane, s/n, 14884-900 Jaboticabal-SP, <ana.schiavetto@hotmail.com>; ${ }^{3}$ Professor, Dr., Bolsista CNPq, Dep. de Ciências Exatas, UNESP, Jaboticabal-SP, <perecin@fcav.unesp.br>; ${ }^{4}$ Professor, Pesquisador, Dr., IAC/Centro de Cana, Rod. Anel Viário Contorno Sul, Km 321, Caixa Postal 206, 14001-970 Ribeirão Preto-SP, <azania@iac.sp.gov.br>; ${ }^{5}$ Mestrando em Agricultura Tropical e Subtropical - Tecnologia da Produção Agrícola, Instituto Agronômico de Campinas - IAC, <fabriciozera@gmail.com>; ${ }^{6}$ PqC Voluntária, Dra., IAC/Centro de Cana, <andrea.azania@hotmail.com>; ${ }^{7}$ Graduanda em Agronomia - Centro Universitário Moura Lacerda, Estagiária de iniciação científica - área de plantas daninhas, IAC/Centro de Cana, <ca.lorenzato@hotmail.com>. 
borders. To check whether the mean differences (TP-TH) did not differ from zero, the following statistics was used: $t=(T P-T H) /\left((Q M R e s / n)^{1 / 2}\right.$ or, equivalently, the least significant difference of zero, dmsO (TP TH) $=\left|t /\left((Q M R e s / n)^{1 / 2}\right)\right|$, where QMRes is the mean square analysis of variance (with 47 degrees of freedom and $p<0.05, t=2$ ), $n$ represents the number of repetitions of their average, 2 to compare the herbicides in a single cultivar, and $2 \times 6=12$ to compare the overall mean of the cultivars. According to the methodology proposed, the cultivars were tolerant to the association of herbicides without any negative effect on yield and quality of the final raw material, although there were initial differences between the cultivars related to toxicity, chlorophyll content, and plant height.

Keywords: selectivity, Saccharum spp., statistics, association of herbicides.

\section{INTRODUÇÃO}

No setor sucroalcooleiro, mesmo tendo o conhecimento de que os cultivares apresentam respostas distintas de tolerância a herbicidas (Oliveira et al., 2004; Ferreira et al., 2005), os programas de melhoramento genético focam a seleção dos novos genótipos principalmente para fatores edafoclimáticos, pragas e doenças (Matsuoka et al., 2005), não considerando a tolerância dos cultivares. A escassez de recursos humanos e de material para propagação, o longo ciclo da cultura e as extensas áreas experimentais são as principais justificativas para a ausência da informação. Segundo Montório et al. (2009), o tamanho da área experimental do delineamento em blocos ao acaso na cultura conduz o pesquisador a erros experimentais, devido à dificuldade imposta pela distância na coleta das variáveis.

As propostas metodológicas que diminuam a área experimental e não comprometam a precisão das variáveis podem servir de estímulo aos estudos de tolerância de cultivares. A utilização do delineamento em blocos aumentados nos programas de melhoramento genético de batata (Bearzoti et al., 1997) e feijão (Souza et al., 2000) é um exemplo de minimização do espaço físico do experimento, em razão de permitir o acréscimo de tratamentos sem repetição nos blocos e a consequente diminuição na área do experimento.

Entretanto, o aumento quantitativo das testemunhas é o artifício mais observado na literatura para melhorar a precisão experimental, porém ele leva ao aumento da área experimental. Constantin (1996), ao propor o uso de uma testemunha para cada parcela tratada com herbicida dentro de cada bloco, observou melhoria na precisão experimental. Giordani et al. (2000), quando adotaram a técnica, obtiveram sucesso no estudo da seletividade de herbicidas em alface. Para Ribeiro et al. (2001), as testemunhas pareadas não reduziram a precisão ao compararem cultivares e linhagens de feijoeiro. Azania et al. (2005), estudando a tolerância da cana-deaçúcar a herbicidas, também constataram a eficiência das testemunhas laterais.

A técnica das testemunhas intercalares também é eficiente porque permite ajustar os valores observados, a partir de valores de parcelas próximas, contendo testemunhas espaçadas sistematicamente na área (TownleySmith \& Hurd, 1973). Segundo Mak et al. (1978), ao estudarem linhagens de cevada, o método possui precisão semelhante à dos métodos de médias móveis e delineamento em látice. Contudo, a técnica das testemunhas pareadas apresenta o aumento da área experimental como a desvantagem do método.

Ao se observar a dificuldade em associar a minimização da área mantendo a precisão experimental, formulou-se a hipótese de que os cultivares de cana-de-açúcar podem ter a tolerância aos herbicidas estudada considerando a diferença entre parcelas testemunhas e tratadas, a partir de um reduzido número de repetições. Para confirmação da hipótese, o presente trabalho teve como objetivo estudar a tolerância de cultivares RB855453, RB845257, SP90-3414, SP90-1638, SP89-1115, SP81-3250, IAC91-2218 e IAC91-5155 de canade-açúcar em pós-emergência inicial quanto à aplicação dos herbicidas sulfentrazone + diuron + hexazinone, metsulfuron-methyl + sulfentrazone, diuron + hexazinone + clomazone, metribuzin + diuron + hexazinone, 
diuron + hexazinone + MSMA e ametryn + trifloxysulfuron-sodium + diuron + hexazinone, analisada pela diferença dos tratamentos com a testemunha pareada, considerando número mínimo de repetições.

\section{MATERIAL E MÉTODOS}

O experimento foi instalado no município de Pradópolis-SP, em soqueiras de sexto corte, no período entre dezembro de 2007 e julho de 2008. O solo da área experimental foi classificado como Latossolo Vermelho de classe textural argilosa $(44,45 \%$ de argila, $23,21 \%$ de silte e $32,34 \%$ de areia), tendo as seguintes características químicas: $\mathrm{pH}$ $(4,8)$, matéria orgânica $\left(37 \mathrm{~g} \mathrm{dm}^{-3}\right)$, fósforo $\left(0,65 \mathrm{mg} \mathrm{dm}^{-3}\right)$, potássio $\left(1,9 \mathrm{mmol}_{\mathrm{c}} \mathrm{dm}^{-3}\right)$, cálcio $\left(38,4 \mathrm{mmol}_{\mathrm{c}} \mathrm{dm}^{-3}\right)$, magnésio $\left(5,4 \mathrm{mmol}_{\mathrm{c}} \mathrm{dm}^{-3}\right)$, CTC $\left(88,5 \mathrm{mmol}_{\mathrm{c}} \mathrm{dm}^{-3}\right)$ e V $(52,22)$. As soqueiras, antes da aplicação dos herbicidas, foram adubadas com $490 \mathrm{~kg} \mathrm{ha}^{-1}$ da formulação 21-00-21.

O delineamento experimental foi em blocos ao acaso, utilizando-se o esquema fatorial $8 \times 6 \mathrm{com}$ testemunhas pareadas, com duas repetições, na tentativa de minimizar a área experimental. As parcelas foram constituídas de seis linhas de cana-de-açúcar de $15 \times 1,50 \mathrm{~m}$; uma linha central foi tratada com herbicida (TH), uma foi deixada como bordadura e outra linha central constituiu a parcela testemunha pareada (TP), totalizando 22,5 $\mathrm{m}^{-2}$ de área útil para as linhas TH e TP; as demais linhas foram usadas como bordaduras. Para efeito de avaliação, considerou-se a diferença entre a testemunha pareada e os tratamentos herbicidas (TP-TH).

Os cultivares RB855453, RB845257, SP813250, SP90-3414, SP90-1638, SP89-1115, IAC91-2218 e IAC91-5155 constituíram o primeiro fator e foram escolhidos por serem indicados para cultivo nas condições ambientais do Centro-Sul do Brasil, de acordo com os conceitos de qualificação ambiental utilizados nos programas de melhoramento. Os herbicidas, constituintes do segundo fator, foram escolhidos por serem amplamente utilizados na cultura, e suas associações constituíram os tratamentos químicos: T1 - sulfentrazone (500 $\left.\mathrm{g} \mathrm{ha}^{-1}\right)+$ diuron $\left(842,4 \mathrm{~g} \mathrm{ha}^{-1}\right)+$ hexazinone (237,6 $\left.\mathrm{g} \mathrm{ha}^{-1}\right)$; T2 - metsulfuron-methyl
(6 $\left.\mathrm{g} \mathrm{ha}^{-1}\right)+$ sulfentrazone (750 $\left.\mathrm{g} \mathrm{ha}^{-1}\right)$; T3 diuron $\left(842,4 \mathrm{~g} \mathrm{ha}^{-1}\right)+$ hexazinone $\left(237,6 \mathrm{~g} \mathrm{ha}^{-1}\right)$ + clomazone (900 $\mathrm{g} \mathrm{ha}^{-1}$ ); T4 - metribuzin $\left(1.920 \mathrm{~g} \mathrm{ha}^{-1}\right)+$ diuron $\left(842,4 \mathrm{~g} \mathrm{ha}^{-1}\right)+$ hexazinone $\left(237,6 \mathrm{~g} \mathrm{ha}^{-1}\right)$; T5 - diuron $\left(1.599 \mathrm{~g} \mathrm{ha}^{-1}\right)+$ hexazinone $\left(201 \mathrm{~g} \mathrm{ha}^{1}\right)+$ MSMA (360 $\left.\mathrm{g} \mathrm{ha}^{-1}\right)$; e T6 - ametryn $\left(1.097,25 \mathrm{~g} \mathrm{ha}^{-1}\right)+$ trifloxysulfuron-sodium $\left(27,75 \mathrm{~g} \mathrm{ha}^{-1}\right)+$ diuron (702 $\left.\mathrm{g} \mathrm{ha}^{-1}\right)+$ hexazinone (198 $\left.\mathrm{g} \mathrm{ha}^{-1}\right)$, cada qual com a testemunha pareada, que foram capinadas e mantidas com ausência de plantas daninhas.

Os herbicidas foram aplicados em condição de pós-emergência inicial da cultura, no dia 28/12/2007, quando as plantas apresentaram altura média de aproximadamente $38 \mathrm{~cm}$. Foi utilizado pulverizador costal pressurizado $\left(\mathrm{CO}_{2}\right)$ munido de barra com duas pontas, modelo TT $110.02 \mathrm{VS}$, espaçadas de $50 \mathrm{~cm}$, que, regulado a 30 psi de pressão, proporcionou volume de calda de $200 \mathrm{~L} \mathrm{ha}^{-1}$. No momento da aplicação a velocidade do vento oscilou entre 0,5 e $4,6 \mathrm{~km} \mathrm{~h}^{-1}$, a temperatura média do ar foi de $30{ }^{\circ} \mathrm{C}$, havia $50 \%$ de nebulosidade e a umidade relativa do ar foi de $52,6 \%$.

Foram avaliados o teor relativo de clorofila total, a eficiência fotoquímica do fotossistema (FS II) e os sintomas de intoxicação na parte aérea das plantas aos 15 e 50 dias após aplicação (DAA), nas linhas TP e TH de cada parcela. O teor relativo de clorofila foi determinado na folha +3 de seis plantas escolhidas ao acaso, fornecida pela leitura direta do clorofilômetro portátil (modelo SPAD 502, Minolta); a eficiência fotoquímica do FS II também foi determinada na folha +3 de seis plantas, pela leitura direta da razão da fluorescência (Fv/Fm), determinada com fluorômetro portátil (PEA - Plant Efficiency Analyser, Hansatech), após 20 minutos de adaptação no escuro. Os sintomas visuais de intoxicação foram avaliados observando-se a sintomatologia na parte aérea das plantas, atribuindo-se nota a partir de uma escala percentual, variando entre 0 e 100, na qual 0 corresponde à ausência de injúrias e 100 à morte das plantas.

Foram realizadas também avaliações de altura $(\mathrm{cm})$ e estande $\left(\right.$ colmos $\left.\mathrm{m}^{-1}\right)$ aos $30 \mathrm{e}$ 180 DAA. Para a altura, tomaram-se dez plantas ao acaso, medindo-se a distância do solo até a lígula da primeira folha aberta; para o 
estande da cultura, foram contados todos os colmos nas linhas TP e TH.

Por ocasião da colheita, aos 210 DAA, foram avaliados a produção de colmos (t ha $\left.{ }^{-1}\right)$, o diâmetro médio dos colmos e a qualidade tecnológica dos colmos da cultura. A produção foi estimada segundo metodologia de Landell et al. (1995). Nas linhas TP e TH, em um ponto ao acaso foram despontados dez colmos sequencialmente, obtendo-se os diâmetros médios; em laboratório de análise de sacarose, foram obtidos os teores de brix do caldo, pol do caldo, fibra da cana e o açúcar total recuperado do caldo (ATR), em kg t cana ${ }^{-1}$, determinado segundo o sistema de pagamento de cana pelo teor de sacarose vigente (Consecana, 2006).

Os atributos avaliados foram analisados pela diferença TP-TH. Inicialmente foram feitas análises de variância, segundo o delineamento utilizado (fatorial $8 \times 6$, em dois blocos), que permitem verificar efeitos da igualdade dessas diferenças entre os cultivares, os tratamentos químicos (herbicidas) e de interações.

Essas análises fornecem também o quadrado médio do resíduo (QMRes), que estima a variância ambiental associada às diferenças (TP-TH) e permite a construção de um teste $\mathrm{t}$ para verificar se as diferenças médias (TP-TH) podem ser zero, ou seja, usa-se: $\mathrm{t}=(\mathrm{TP}-\mathrm{TH}) /\left((\mathrm{QMRes} / \mathrm{n})^{1 / 2}\right)$, ou forma equivalente, e a diferença mínima significativa com zero, dmsO (TP-TH) $=\left|\mathrm{t} /\left((\mathrm{QMRes} / \mathrm{n})^{1 / 2}\right)\right|$, em que QMRes é o quadrado médio da análise de variância e $\mathrm{n}$ representa o número de repetições da respectiva média. Essa é uma forma interessante e mais objetiva que a usada por Azania et al. (2005) para avaliar as diferenças entre as testemunhas pareadas.

No caso do delineamento utilizado, o grau de liberdade associado ao QMRes é 47, resultando que o valor de t bilateral $(p=0,05)$ é de aproximadamente 2 . Portanto, para a comparação de um herbicida em um cultivar, a diferença (TP-TH) será diferente de zero se superar (em valor absoluto), $\mathrm{dmsO}(\mathrm{i})=\mid 2$ $(\text { QMRes } / 2)^{1 / 2} \mid$; para a diferença da média do cultivar (seis herbicidas), dms $(\mathrm{m})=\mid 2$ $(\text { QMRes } /(6.2))^{1 / 2} \mid$. Os valores do denominador referem-se ao número de blocos (2) e ao número de herbicidas (6).

\section{RESULTADOS E DISCUSSÃO}

Em relação à fitointoxicação, observaramse cloroses e amarelecimento nas folhas da cultura aos 15 DAA e ausência de sintomas aos 50 DAA. Constata-se, na Figura 1, que as notas de fitointoxicação variaram de 5 a $35 \%$ nas plantas dos cultivares estudados, sendo o tratamento T3 - diuron $\left(842,4 \mathrm{~g} \mathrm{ha}^{-1}\right)$ + hexazinone $\left(237,6 \mathrm{~g} \mathrm{ha}^{-1}\right)+$ clomazone (900 $\mathrm{g} \mathrm{ha}^{-1}$ ) o que originou as maiores notas de fitointoxicação em todos os cultivares. Os cultivares SP90-3414 e SP89-5155 apresentaram as menores notas para este tratamento: 20 e $20 \%$, respectivamente; e o cultivar RB845257, a maior nota: 35\%. No experimento, essas avaliações foram importantes porque a maioria dos herbicidas utilizados possui como mecanismo de ação a inibição de alguma estrutura no aparato fotossintético, a exemplo dos herbicidas que interferem no fotossistema II ou na inibição do caroteno (Rodrigues \& Almeida, 2005).

Ao analisar as médias das notas atribuídas a cada tratamento dentro de cada cultivar, observou-se que o cultivar SP891115 apresentou os sintomas de intoxicação mais intensos (20,3\%), e o SP90-3414, os de menor intensidade $(9,8 \%)$. A associação dos herbicidas T3- diuron $\left(842,4 \mathrm{~g} \mathrm{ha}^{-1}\right)+$ hexazinone $\left(237,6 \mathrm{~g} \mathrm{ha}^{-1}\right)+$ clomazone (900 $\mathrm{g} \mathrm{ha}{ }^{1}$ ) foi a que proporcionou os maiores sintomas de intoxicação $(26,4 \%)$, e o T1 - sulfentrazone $\left(500 \mathrm{~g} \mathrm{ha}^{-1}\right)+$ diuron $\left(842,4 \mathrm{~g} \mathrm{ha}^{-1}\right)+$ hexazinone $\left(237,6 \mathrm{~g} \mathrm{ha}^{-1}\right)$, os menores sintomas $(9,9 \%)$.

Os dados obtidos em campo, embora oriundos de específicas condições edafoclimáticas, doses, cultivares e características fisicoquímicas dos herbicidas, corroboram os de trabalhos observados na literatura. Resultados similares foram encontrados por Souza et al. (2009), ao utilizarem a mesma escala visual no estudo de tolerância de cultivares de cana-de-açúcar, porém com delineamento estatístico tradicional com quatro repetições. Esses autores observaram notas atribuídas aos sintomas de intoxicação não superiores a $20 \%$ aos 14 dias da aplicação, com recuperação da cultura a partir dos 60 dias. 

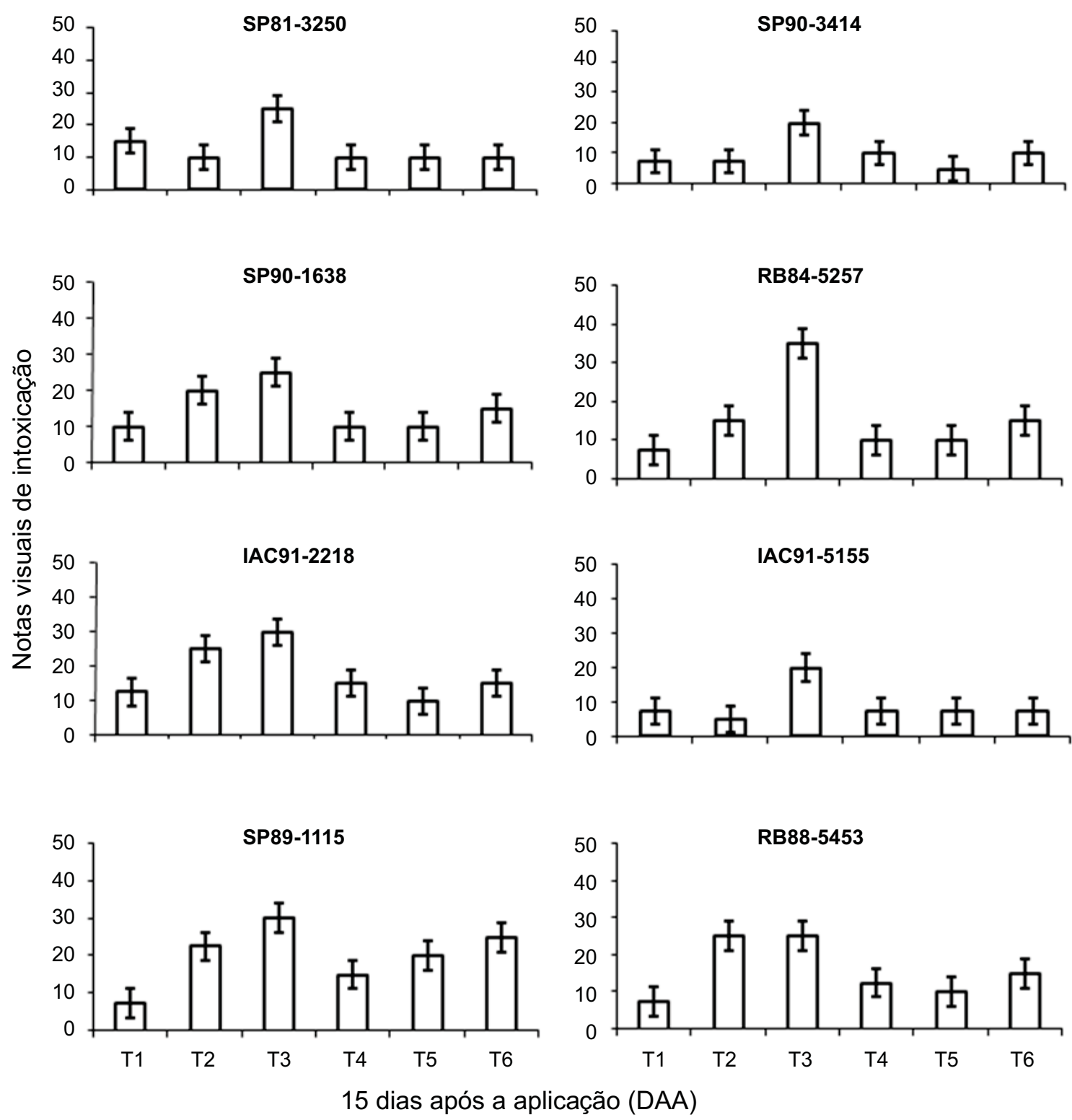

Erro-padrão: $\hat{I}=(\sqrt{ }($ QMRes $) / \sqrt{ }($ número de repetições $))$. T1= sulfentrazone $\left(500 \mathrm{~g} \mathrm{ha}^{-1}\right)+$ diuron $\left(842,4 \mathrm{~g} \mathrm{ha}^{-1}\right)+$ hexazinone $\left(237,6 \mathrm{~g} \mathrm{ha}{ }^{-1}\right)$; $\mathrm{T} 2=$ metsulfuron-methyl $\left(6 \mathrm{~g} \mathrm{ha}^{-1}\right)+$ sulfentrazone $\left(750 \mathrm{~g} \mathrm{ha}^{-1}\right)$; $\mathrm{T} 3=$ diuron $\left(842,4 \mathrm{~g} \mathrm{ha}^{1}\right)+$ hexazinone $\left(237,6 \mathrm{~g} \mathrm{ha}^{-1}\right)+$ clomazone $^{-1}$ $\left(900 \mathrm{~g} \mathrm{ha}^{-1}\right)$; T4= metribuzin $\left(1.920 \mathrm{~g} \mathrm{ha}^{-1}\right)+$ diuron $\left(842,4 \mathrm{~g} \mathrm{ha}^{-1}\right)+$ hexazinone $\left(237,6 \mathrm{~g} \mathrm{ha}^{-1}\right)$; $\mathrm{T} 5=$ diuron $\left(1.599 \mathrm{~g} \mathrm{ha}^{1}\right)+$ hexazinone $\left(201 \mathrm{~g} \mathrm{ha}^{-1}\right)+$ MSMA $\left(360 \mathrm{~g} \mathrm{ha}^{-1}\right)$; T6= ametryn $\left(1.097,25 \mathrm{~g}^{\mathrm{ha}}{ }^{1}\right)+\operatorname{trifloxysulfuron-sodium~}\left(27,75 \mathrm{~g} \mathrm{ha}^{-1}\right)+$ diuron $\left(702 \mathrm{~g} \mathrm{ha}^{-1}\right)$ + hexazinone (198 $\left.\mathrm{g} \mathrm{ha}^{-1}\right)$.

Figura 1 - Porcentagem de notas atribuídas aos sintomas de intoxicação em cana-de-açúcar tratada com diferentes associações de herbicidas. Instituto Agronômico, 2007/2008.

O teor relativo de clorofila (Tabela 1) entre os cultivares foi mais reduzido aos 15 DAA, enquanto aos 50 DAA poucas interferências foram observadas. Na primeira época de avaliação, o menor valor observado $(34,63)$ foi no cultivar SP89-1115 tratado com T5-diuron
(1.599 $\left.\mathrm{g} \mathrm{ha}^{-1}\right)+$ hexazinone (201 $\left.\mathrm{g} \mathrm{ha}^{-1}\right)+$ MSMA (360 $\left.\mathrm{g} \mathrm{ha}^{-1}\right)$, e o maior valor $(47,38)$ foi constatado na testemunha do cultivar RB855453. Ferreira et al. (2010) também observaram redução no teor de clorofila, cultivar SP80-3280, fazendo uso de diuron + hexazinone e MSMA. 
Tabela 1 - Teor relativo de clorofila total em diferentes cultivares de cana-de-açúcar aos 15 e 50 dias após tratamento, nos tratamentos herbicidas (TH), testemunha pareada (TP) e diferença (TP-TH). Instituto Agronômico, 2007/2008

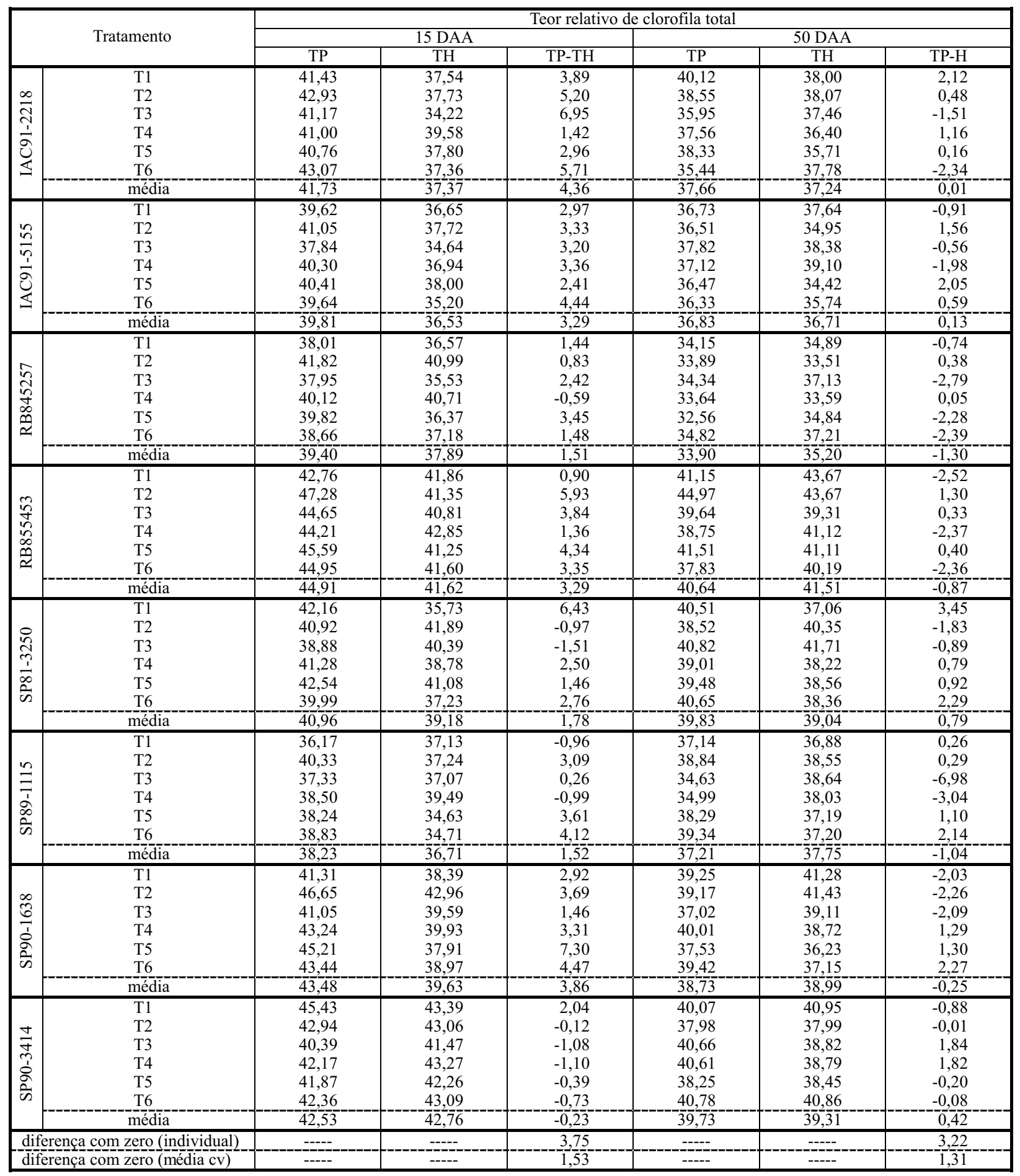

** Significância a 1\%; ns: não significativo; dados médios de duas repetições. T1 = sulfentrazone $\left(500 \mathrm{~g}\right.$ ha $\left.{ }^{-1}\right)+$ diuron $\left(842,4 \mathrm{~g} \mathrm{ha}^{-1}\right)+$ hexazinone $\left(237,6 \mathrm{~g} \mathrm{ha}^{-1}\right) ; \mathrm{T} 2=$ metsulfuron-methyl $\left(6 \mathrm{~g} \mathrm{ha}^{-1}\right)+$ sulfentrazone $\left(750 \mathrm{~g} \mathrm{ha}^{-1}\right) ; \mathrm{T} 3=$ diuron $\left(842,4 \mathrm{~g}\right.$ ha $\left.{ }^{1}\right)+$ hexazinone $\left(237,6 \mathrm{~g} \mathrm{ha}^{-1}\right)+$ clomazone $\left(900 \mathrm{~g} \mathrm{ha}^{-1}\right)$; $\mathrm{T} 4=$ metribuzin $\left(1.920 \mathrm{~g} \mathrm{ha}^{1}\right)+\operatorname{diuron}\left(842,4 \mathrm{~g} \mathrm{ha}^{-1}\right)+$ hexazinone $\left(237,6 \mathrm{~g} \mathrm{ha}^{-1}\right)$; T5 = diuron $\left(1.599 \mathrm{~g} \mathrm{ha}^{-1}\right)+$ hexazinone $\left(201 \mathrm{~g} \mathrm{ha}^{-1}\right)+$ MSMA $\left(360 \mathrm{~g} \mathrm{ha}^{-1}\right)$; T6 = ametryn $\left(1.097,25 \mathrm{~g} \mathrm{ha}^{1}\right)+$ trifloxysulfuron-sodium $\left(27,75 \mathrm{~g} \mathrm{ha}^{-1}\right)+$ diuron $\left(702 \mathrm{~g} \mathrm{ha}^{-1}\right)+$ hexazinone $\left(198 \mathrm{~g} \mathrm{ha}^{-1}\right)$. 
A média da diferença entre TP-TH nos cultivares permitiu observar a real redução do teor relativo de clorofila em cada cultivar, pois, quando seus valores foram superiores ao limite estatístico de 1,53 (diferença com zero), a diferença foi considerada representativa. Assim, as médias das diferenças entre TP e TH nos cultivares IAC91-2218, IAC91-5155, RB855453 e SP90-1638 sugerem redução nos teores de clorofila, correspondente a 10,45; 8,$26 ; 7,33$; e $8,88 \%$, respectivamente.

A associação entre T6- ametryn $\left(1.097,25 \mathrm{~g} \mathrm{ha}^{-1}\right)+$ trifloxysulfuron-sodium $\left(27,75 \mathrm{~g} \mathrm{ha}^{1}\right)+$ diuron $\left(702 \mathrm{~g} \mathrm{ha}^{-1}\right)+$ hexazinone $\left(198 \mathrm{~g} \mathrm{ha}^{-1}\right)$ foi o tratamento que mais interferiu no teor relativo de clorofila dos cultivares IAC91-2218, IAC91-5155, SP89-1115 e SP901638. Os valores de TP-TH do referido tratamento superaram o limite de 3,75 (diferença com zero para herbicida), destacando-o como o herbicida que reduziu o teor de clorofila em $50 \%$ dos cultivares estudados.

Nos cultivares, a razão da fluorescência da clorofila $a$ não foi prejudicada por nenhum herbicida (dados não apresentados). Entretanto, houve prejuízo em relação ao desenvolvimento dos cultivares, particularmente quanto a altura, estande e produtividade.

Aos 30 DAA, observou-se, de acordo com as médias dos cultivares, que todos foram prejudicados quanto à altura, pois as diferenças TP-TH foram superiores aos 3,68 da diferença com zero (média cultivar). O RB845257, aos 30 DAA, apresentou redução de $32,7 \%$ com o T3 = diuron $\left(842,4 \mathrm{~g} \mathrm{ha}^{-1}\right)+$ hexazinone $\left(237,6 \mathrm{~g} \mathrm{ha}^{-1}\right)+$ clomazone (900 $\mathrm{g} \mathrm{ha}^{-1}$ ). Ferreira et al. (2010), ao estudarem a tolerância de cultivares de cana-de-açúcar a estresse por herbicidas, observaram que diuron + hexazinone ocasionaram redução em altura cultivar CTC5.

Alguns cultivares apresentaram prejuízos com os tratamentos envolvendo a mistura pronta de ametryn+trifloxysulfuronsodium, devido à paralisação do crescimento, pois, segundo Ferreira et al. (2005), a associação desses ingredientes ativos causa essa interferência. Na literatura, estudos demonstraram que os herbicidas ametryn+ trifloxysulfuron-sodium e sulfentrazone reduziram a altura de cultivares de cana-de-açúcar
(Ferreira et al., 2005; Barela \& Christoffoleti, 2006).

Aos 180 DAA, os cultivares ainda apresentavam redução de altura em relação à TP, exceto RB845257 e SP90-3414, por terem apresentado médias superiores a 5,77 (Tabela 2). Em estudos de seletividade, Montório et al. (2009) também constataram redução em altura das plantas de cana-deaçúcar com a utilização de oxyfluorfen e diuron na pré-emergência da cultura, ou seja, com o uso minimizado de área (testemunha pareada), foram observadas reduções semelhantes às verificadas quando avaliado em um delineamento tradicional, com quatro repetições.

Galon et al. (2009), ao estudarem a tolerância de novos genótipos de cana-de-açúcar a herbicidas, observaram aos 360 DAT que os genótipos também apresentaram redução em relação à testemunha, exceto RB867515 e SP80-1816, os quais mostraram maior altura de plantas quando comparados com a testemunha capinada.

Nas avaliações de estande para cada tratamento herbicida (Tabela 3) a diferença TP-TH aos 30 DAA, constatou-se que a mistura $\mathrm{T} 1=$ sulfentrazone $\left(500 \mathrm{~g} \mathrm{ha}^{-1}\right)+$ diuron $\left(842,4 \mathrm{~g} \mathrm{ha}^{-1}\right)+$ hexazinone $\left(237,6 \mathrm{~g} \mathrm{ha}^{-1}\right)$ reduziu o estande dos cultivares IAC91-2218 (16,67\%) e SP81-3250 (17,65\%), possivelmente, pelo fato de eles terem sido mais afetados pelos herbicidas nas avaliações do teor relativo de clorofila total (Tabela 1). Constantin (2001) relatou que o diclosulam, embora não utilizado no estudo, também apresentou tendência em reduzir o perfilhamento e a altura da canade-açúcar.

Aos 180 DAA, não foram mais observadas reduções no estande de todos os cultivares $\mathrm{e}$ tratamentos, analisados com diferença com zero (média de cultivar e herbicida dentro de cultivar), indicando recuperação dos cultivares sob a influência dos herbicidas. Souza et al. (2009) também não encontraram perdas ao estudarem a tolerância a herbicidas para os cultivares IACSP94-2094, IACSP94-2101, IACSP93-3046, IACSP94-4004, RB72454 e IAC86-2480, indicando apenas diferenças inerentes à própria genética do cultivar.

Na ocasião da colheita, aos 210 DAA, não houve redução na produtividade dos colmos em 
Tabela 2 - Altura (cm) em diferentes cultivares de cana-de-açúcar aos 30 e 180 dias após tratamento, nos tratamentos herbicidas (TH), testemunha pareada (TP) e diferença (TP-H). Instituto Agronômico, 2007/2008

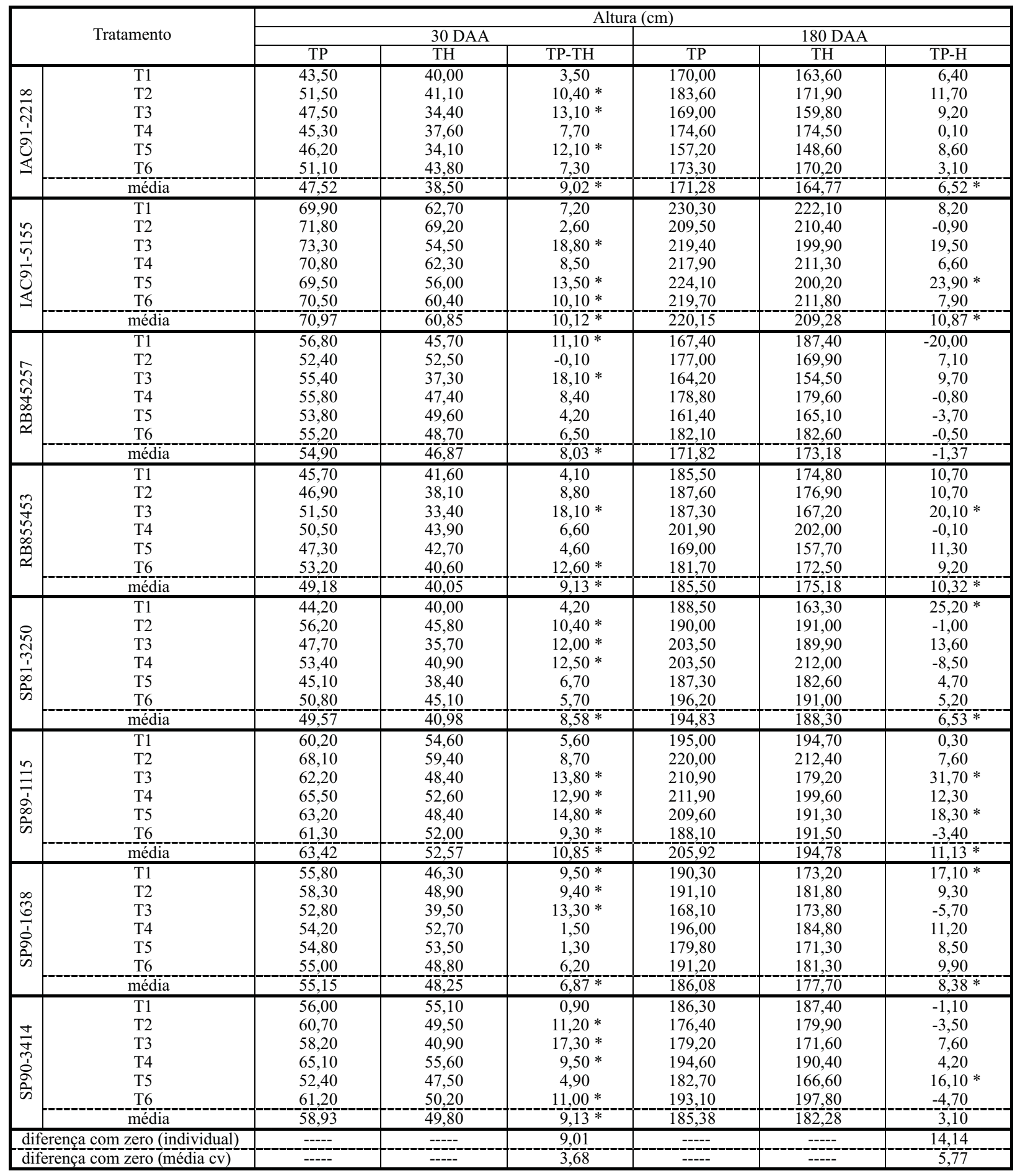

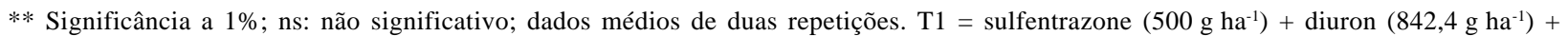
hexazinone $\left(237,6 \mathrm{~g} \mathrm{ha}^{-1}\right)$; T2 = metsulfuron-methyl $\left(6 \mathrm{~g} \mathrm{ha}^{-1}\right)+$ sulfentrazone $\left(750 \mathrm{~g} \mathrm{ha}^{-1}\right)$; $\mathrm{T} 3=$ diuron $\left(842,4 \mathrm{~g}\right.$ ha $\left.{ }^{1}\right)+$ hexazinone $\left(237,6 \mathrm{~g} \mathrm{ha}^{-1}\right)+$ clomazone $\left(900 \mathrm{~g} \mathrm{ha}^{-1}\right)$; $\mathrm{T} 4=$ metribuzin $\left(1.920 \mathrm{~g} \mathrm{ha}^{1}\right)+\operatorname{diuron}\left(842,4 \mathrm{~g} \mathrm{ha}^{-1}\right)+$ hexazinone $\left(237,6 \mathrm{~g} \mathrm{ha} \mathrm{a}^{-1}\right)$; $\mathrm{T} 5=\operatorname{diuron}^{-1}$ $\left(1.599 \mathrm{~g} \mathrm{ha}^{-1}\right)+$ hexazinone $\left(201 \mathrm{~g} \mathrm{ha}^{-1}\right)+$ MSMA $\left(360 \mathrm{~g} \mathrm{ha}^{-1}\right)$; T6 = ametryn $\left(1.097,25 \mathrm{~g} \mathrm{ha}^{1}\right)+$ trifloxysulfuron-sodium $\left(27,75 \mathrm{~g}\right.$ ha $\left.{ }^{-1}\right)+$ diuron (702 $\left.\mathrm{g} \mathrm{ha}^{-1}\right)+$ hexazinone (198 $\left.\mathrm{g} \mathrm{ha}^{-1}\right)$. 
Tolerância de cana-de-açúcar a herbicidas avaliada pela diferença ...

Tabela 3 - Estande (colmos $\mathrm{m}^{-1}$ ) em diferentes cultivares de cana-de-açúcar aos 30 e 180 dias após tratamento, nos tratamentos herbicidas (TH), testemunha pareada (TP) e diferença (TP-H). Instituto Agronômico, 2007/2008

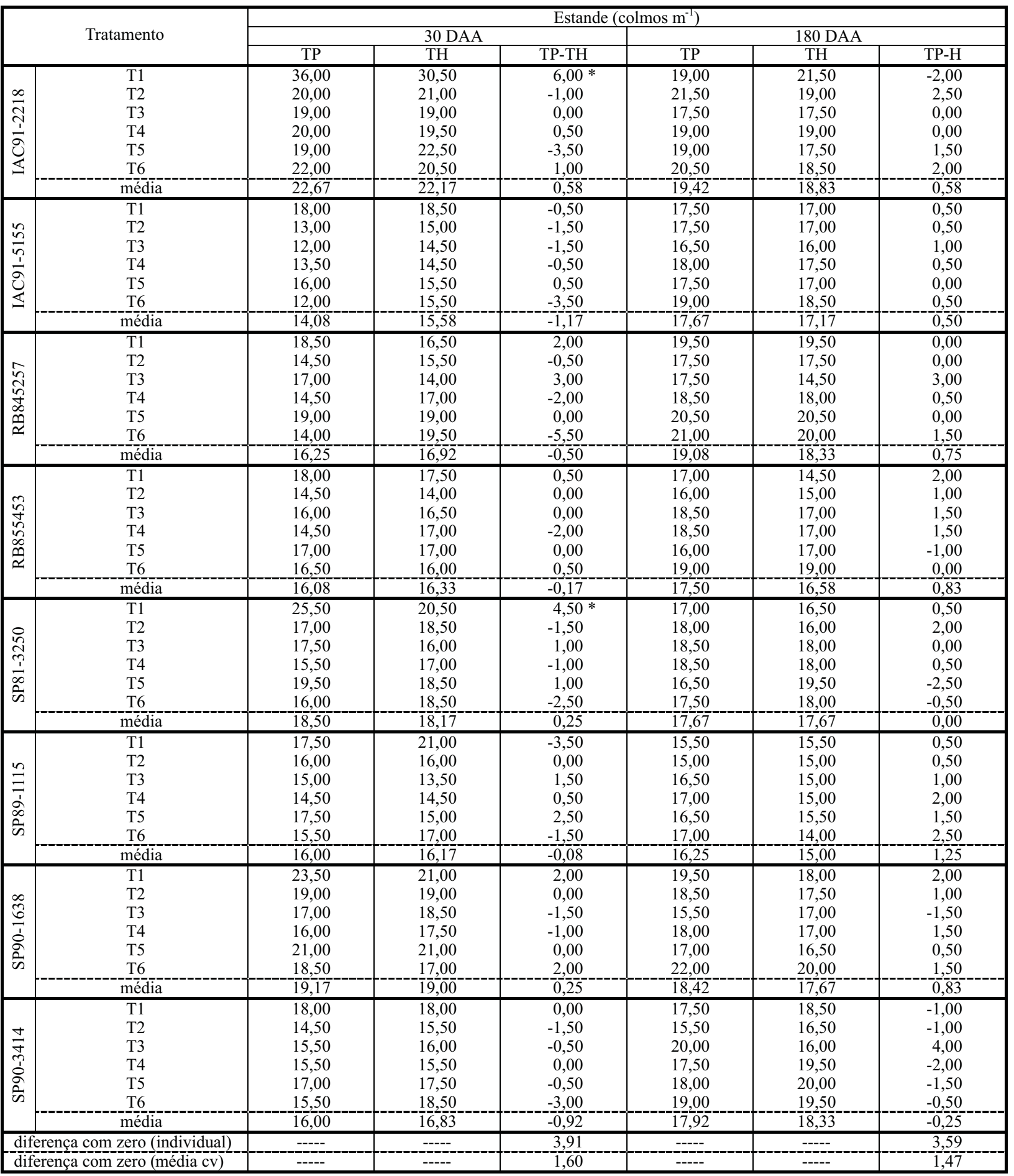

** Significância a $1 \%$; ns: não significativo; dados médios de duas repetições. T1 $=$ sulfentrazone $\left(500 \mathrm{~g} \mathrm{ha}^{-1}\right)+$ diuron $\left(842,4 \mathrm{~g} \mathrm{ha}^{-1}\right)+$ hexazinone $\left(237,6 \mathrm{~g} \mathrm{ha}^{-1}\right)$; T2 = metsulfuron-methyl $\left(6 \mathrm{~g} \mathrm{ha}^{-1}\right)+$ sulfentrazone $\left(750 \mathrm{~g} \mathrm{ha}^{-1}\right)$; $\mathrm{T} 3=$ diuron $\left(842,4 \mathrm{~g}\right.$ ha $\left.{ }^{1}\right)+$ hexazinone $\left(237,6 \mathrm{~g} \mathrm{ha}^{-1}\right)+$ clomazone $\left(900 \mathrm{~g} \mathrm{ha}^{-1}\right)$; $\mathrm{T} 4=$ metribuzin $\left(1.920 \mathrm{~g} \mathrm{ha}^{1}\right)+\operatorname{diuron}\left(842,4 \mathrm{~g} \mathrm{ha}^{-1}\right)+$ hexazinone $\left(237,6 \mathrm{~g} \mathrm{ha} \mathrm{a}^{-1}\right)$; 5 = diuron $\left(1.599 \mathrm{~g} \mathrm{ha}^{-1}\right)+$ hexazinone $\left(201 \mathrm{~g} \mathrm{ha}^{-1}\right)+$ MSMA $\left(360 \mathrm{~g} \mathrm{ha}^{-1}\right)$; T6 = ametryn $\left(1.097,25 \mathrm{~g} \mathrm{ha}^{1}\right)+$ trifloxysulfuron-sodium $\left(27,75 \mathrm{~g}\right.$ ha $\left.{ }^{-1}\right)+$ diuron (702 $\left.\mathrm{g} \mathrm{ha}^{-1}\right)+$ hexazinone (198 $\left.\mathrm{g} \mathrm{ha}^{-1}\right)$. 
Tabela 4 - Produtividade ( $\mathrm{t} \mathrm{ha}^{-1}$ ) e Pol (\% caldo) em diferentes cultivares de cana-de-açúcar aos 210 dias após tratamento, nos tratamentos herbicidas (TH), testemunha pareada (TP) e diferença (TP-H). Instituto Agronômico, 2007/2008

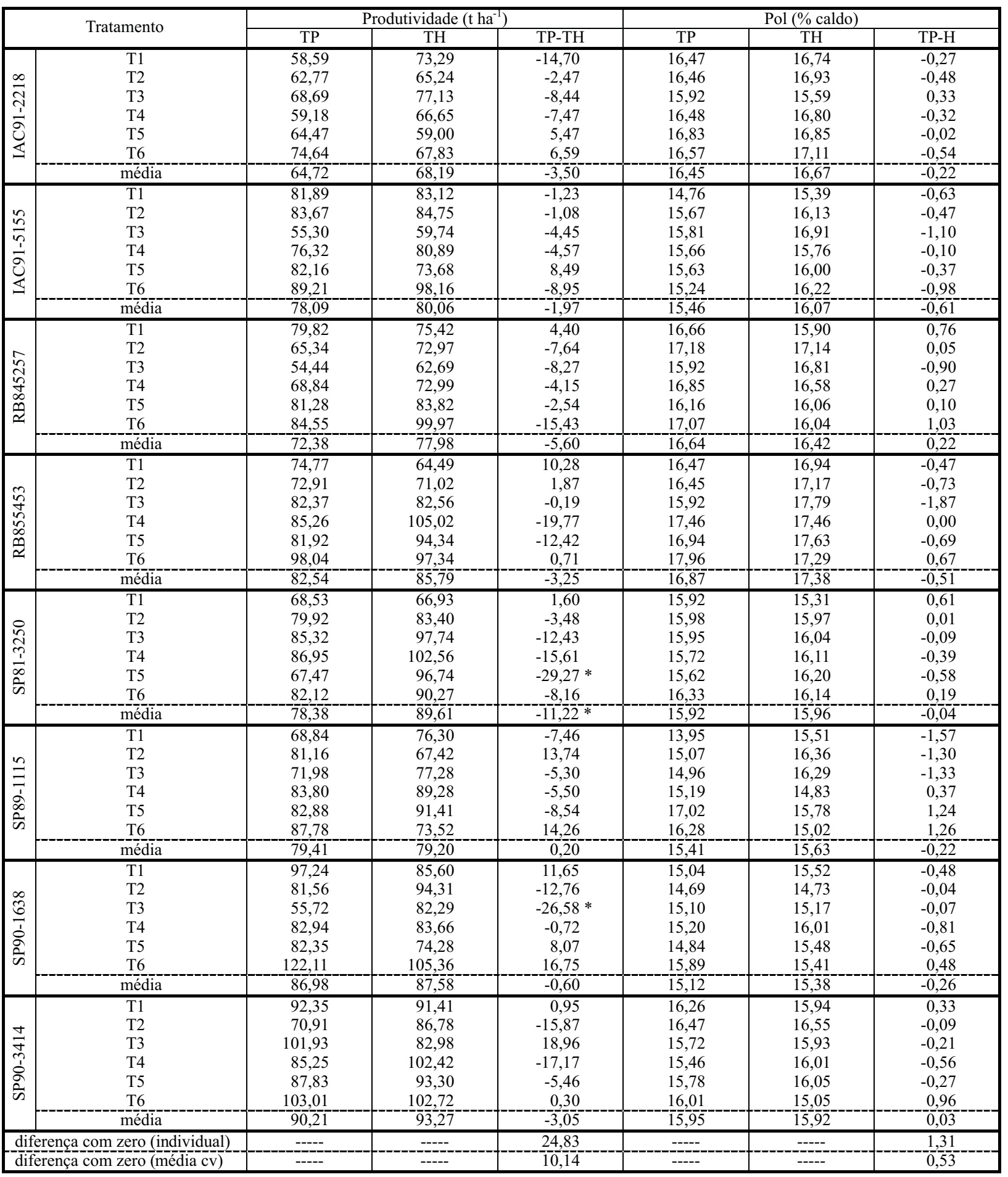

**Significância a $1 \%$; ns: não significativo; dados médios de duas repetições. T1 $=$ sulfentrazone $\left(500 \mathrm{~g} \mathrm{ha}^{-1}\right)+$ diuron $\left(842,4 \mathrm{~g} \mathrm{ha}{ }^{-1}\right)+$ hexazinone $\left(237,6 \mathrm{~g} \mathrm{ha}^{-1}\right)$; T2 = metsulfuron-methyl $\left(6 \mathrm{~g} \mathrm{ha}^{-1}\right)+$ sulfentrazone $\left(750 \mathrm{~g} \mathrm{ha}^{-1}\right)$; $\mathrm{T} 3=$ diuron $\left(842,4 \mathrm{~g}\right.$ ha $\left.{ }^{1}\right)+$ hexazinone $\left(237,6 \mathrm{~g} \mathrm{ha}^{-1}\right)+$ clomazone $\left(900 \mathrm{~g} \mathrm{ha}^{-1}\right)$; $\mathrm{T} 4=$ metribuzin $\left(1.920 \mathrm{~g} \mathrm{ha}^{1}\right)+\operatorname{diuron}\left(842,4 \mathrm{~g} \mathrm{ha}^{-1}\right)+$ hexazinone $\left(237,6 \mathrm{~g} \mathrm{ha} \mathrm{a}^{-1}\right)$; 5 = diuron $\left(1.599 \mathrm{~g} \mathrm{ha}^{-1}\right)+$ hexazinone $\left(201 \mathrm{~g} \mathrm{ha}^{-1}\right)+$ MSMA $\left(360 \mathrm{~g} \mathrm{ha}^{-1}\right)$; T6 = ametryn $\left(1.097,25 \mathrm{~g} \mathrm{ha}^{1}\right)+$ trifloxysulfuron-sodium $\left(27,75 \mathrm{~g}\right.$ ha $\left.{ }^{-1}\right)+$ diuron (702 $\left.\mathrm{g} \mathrm{ha}^{-1}\right)+$ hexazinone (198 $\left.\mathrm{g} \mathrm{ha}^{-1}\right)$. 
relação à TP, pois, ao observar as médias TP-TH, verifica-se valor inferior à diferença com zero (média cultivar e herbicida dentro de cultivar), conforme a Tabela 4. Alguns cultivares apresentaram aumento de produtividade em relação às plantas não tratadas (TP), possivelmente devido a característica inerente à genética do cultivar. $O$ fato de não ter havido perdas com nenhuma das associações entre os herbicidas, ao considerar as médias dos cultivares, é um resultado positivo para o produtor.

Fagliari et al. (2001), ao avaliarem a seletividade de herbicidas aplicados em soqueira de cana-de-açúcar, utilizando testemunhas duplas adjacentes, observaram que o tratamento clomazone + ametryne $(1.000+1.500$ g i.a. ha-1 $)$ alterou a produtividade de colmos da cana-deaçúcar. Essa discordância entre a literatura e os dados observados pode ser atribuída ao material genético em estudo, pois os autores utilizou o cultivar RB835089, diferente daqueles utilizados no presente trabalho. Em outros estudos de produtividade de cana-deaçúcar, encontram-se autores que enfatizam as diferenças entre herbicidas aplicados sobre alguns cultivares, causando ou não perdas de produtividade, a exemplo de Barela \& Christoffoleti (2006) e Ferreira et al. (2005).

Os resultados das análises tecnológicas não mostraram diferenças significativas, ou seja, as diferenças TP-TH apresentaram valor inferior ao estipulado com a diferença com zero (herbicida dentro de cultivar), razão pela qual os dados não foram apresentados. Exceto o cultivar SP89-1115, o qual apresentou aumento no teor de pol\%cana com os tratamentos $\mathrm{T} 1=$ sulfentrazone $\left(500 \mathrm{~g} \mathrm{ha}^{-1}\right)+$ diuron $\left(842,4 \mathrm{~g} \mathrm{ha}^{-1}\right)+$ hexazinone $\left(237,6 \mathrm{~g}^{\text {ha }}{ }^{1}\right)$ e T3 = diuron $\left(842,4 \mathrm{~g} \mathrm{ha}^{-1}\right)+$ hexazinone $\left(237,6 \mathrm{~g} \mathrm{ha}^{-1}\right)+$ clomazone (900 $\left.\mathrm{g} \mathrm{ha}^{1}\right)$, conforme Tabela 4. Isso pode ser explicado pelo fato de a cultura ter se destoxificado das moléculas de herbicidas (Oliveira, 2001), aos 210 DAA, pois o cultivar foi classificado como sensivel ao uso dos herbicidas. Galon et al. (2009), ao trabalharem com os herbicidas ametryn, trifloxysulfuron-sodium e ametryn + trifloxysulfuron-sodium, constataram que a molécula isolada de ametryn ocasionou redução de $18,5 \%$ na produtividade do cultivar RB855156, e o trifloxysulfuron-sodium reduziu em cerca de 12,23 e $17 \%$ a produtividade de açúcar dos cultivares RB72454, RB835486 e RB855156, respectivamente. Já na associação de ametryn + trifloxysulfuron-sodium, Galon et al. (2009), para a mesma variável em estudo, constataram queda de aproximadamente 11,10 e $11 \%$ nos cultivares RB855113, RB855156 e RB925211, respectivamente.

É conveniente ressaltar que, em vários trabalhos encontrados na literatura acerca do uso de herbicidas e tolerância de cultivares de cana-de-açúcar, há relatos mostrando grande diferença entre os cultivares avaliados e que a influência dos herbicidas sobre estes, em especial na qualidade da matéria-prima, deve-se em parte às características intrínsecas dos cultivares utilizados.

Assim, pela metodologia proposta, pode-se concluir que os cultivares foram tolerantes às associações de herbicidas propostas, sem prejuízo final à produtividade e à qualidade da matéria-prima, embora ocorram diferenças iniciais entre cultivares quanto aos sintomas de intoxicação dos herbicidas e à altura das plantas.

\section{AGRADECIMENTOS}

Ao Pesquisador Dr. Marcos Landell (IAC/Cana), pelo apoio em infraestrutura; ao Professor Dr. Pedro Luis da Costa Aguiar Alves (UNESP/FCAV/Jaboticabal), pelo empréstimo dos aparelhos; ao estagiário Igor Vanzela Pizzo, pelo apoio na aplicação dos herbicidas; à Dra. Liliam Silvia Cândido, pelo apoio nas análises estatísticas; ao técnico de apoio Dorival Rodrigues (IAC/Cana), pela ajuda na condução da pesquisa; e à Coordenação de Aperfeiçoamento de Pessoal de Nivel Superior (CAPES), pela concessão da bolsa de estudos à primeira autora.

\section{LITERATURA CITADA}

AZANIA, C. A. M. et al. Seletividade de herbicidas. I utilização do método de testemunhas pareadas em experimentos com cana-de-açúcar. Planta Daninha, v. 23, n. 4, p. 661-667, 2005.

BARELA, J. F.; CHRISTOFFOLETI, P. J. Seletividade de herbicidas aplicados em pré-emergência da cultura da cana-deaçúcar (RB867515) tratada com nematicidas.

Planta Daninha, v. 24, n. 2, p. 371-378, 2006. 
BEARZOTI, E.; PINTO, C. A. B. P.; OLIVEIRA, M. S. Comparação entre métodos estatísticos na avaliação de clones de um programa de melhoramento de batata. Pesq. Agropec. Bras., v. 32, n. 9, p. 877-884, 1997.

CONSELHO DE PRODUTORES DE CANA, AÇÚCAR E ÁLCOOL DE SÃO PAULO - CONSECANA. Manual de instruções. 5.ed. Piracicaba: 2006. 112 p. Disponível em: $<$ http://www.unica.com.br/publicaçoes.com.br>. Acesso em: 18 dez. de 2009.

CONSTANTIN, J. Cana-de-açúcar - seletividade de herbicidas. Correio agrícola. 2.ed. 2001. p. 18-19.

CONSTANTIN, J. Avaliação da seletividade do herbicida halosulfuron à cana-de-açúcar (Saccharum spp.). 1996.

$71 \mathrm{f}$. Tese (Doutorado em Agronomia) - Universidade

Estadual Paulista, Botucatu, 1996.

FAGLIARI, J. R.; OLIVEIRA JÚNIOR, R. S.; CONSTANTIN, J. Métodos de avaliação da seletividade de herbicidas para a cultura da cana-de-açúcar (Saccharum spp.). Acta Sci., v. 23, n. 5, p. 1229-1234, 2001.

FERREIRA, R. R. et al. Tolerância diferencial de variedades de cana-de-açúcar a estresse por herbicidas. Bragantia, v. 69, n. 2, p. 395-404, 2010.

FERREIRA, E. A. et al. Sensibilidade de cultivares de canade-açúcar à mistura trifloxysulfuron-sodim + ametryn.

Planta Daninha, v. 23, n. 1, p. 93-99, 2005.

GALON, L. et al. Influência de herbicidas na qualidade da matéria-prima de genótipos de cana-de-açúcar.

Planta Daninha, v. 27, n. 3, p. 555-562, 2009.

GIORDANI, G. M. R. C. et al. Seletividade de herbicidas aplicados em pré e pós-transplante da cultura da alface. Acta Sci., v. 22, n. 4, p. 985-991, 2000.

LANDELL, M. G. A. Método experimental: Ensaios de competição em cana-de-açúcar. In: MARTINS, L. M.; LANDELL, M. G. A. Conceitos e critérios para avaliação experimental em cana-de-açúcar utilizados no Programa Cana IAC. Pindorama: Instituto Agronômico de Campinas, 1995. p. 2-14.
MAK, C.; HARVEY, B. L.; BERDAHL, J. D. An evaluation of control plots and moving means for erro control in barley nurseries. Crop Sci., v. 18, n. 5, p. 870-873, 1978.

MATSUOKA, S.; GARCIA, A. A. F.; ARIZONO, H. Melhoramento da cana-de-açúcar. In: BORÉM, A. (Ed.). Melhoramento de espécies cultivadas. Viçosa, MG: Universidade Federal de Viçosa, 2005. p. 225-274.

MONTÓRIO, G. A. et al. Seletividade de herbicidas sobre as características de produção da cana-de-açúcar utilizando-se duas testemunhas. Disponível em: <http:// www.upf.br/rbherbicidas/download/RBH226>. Acesso em: 14 dez. de 2009.

OLIVEIRA, M. F. Comportamento de herbicidas no ambiente. In: OLIVEIRA JR., R.S.; CONSTANTIN, J. Plantas daninhas e seu manejo. Guaíba: Agropecuária, 2001. p. 315-362.

OLIVEIRA, R. A. et al. Crescimento e desenvolvimento de três cultivares de cana-de-açúcar, em cana planta, no Estado do Paraná: Taxas de crescimento. Sci. Agr., v. 5, n. 1-2, p. 87-94, 2004.

RIBEIRO, N. D.; STORCK, L.; MELLO, R. M. Bordadura em ensaios de competição de genótipos de feijoeiro relacionados à precisão experimental. Ci. Rural, v. 31, n. 1, p. 13-17, 2001.

RODRIGUES, B. N.; ALMEIDA, F. S. Guia de herbicidas. Londrina: Edição dos Autores, 2005. 648 p.

SOUZA, J. R. et al. Tolerância de cultivares de cana-de-açúcar a herbicidas aplicados em pós-emergência. Bragantia, v. 68, n. 4, p. 941-951, 2009.

SOUZA, E. A.; GERALDI, I. O.; RAMALHO, M. A. P. Alternativas experimentais na avaliação de famílias em programas de melhoramento genético do feijoeiro. Pesq. Agropec. Bras., v. 35, n. 9, p. 1765-1771, 2000.

TOWNLEY-SMITH, T. F.; HURD, E. A. Use of moving means in wheat yield trials. Canadian J. Plant Sci., v. 53, n. 3, p. 447-450, 1973. 